\title{
A ROBUST CONTENT-BASED JPWL TRANSMISSION OVER A REALISTIC MIMO CHANNEL UNDER PERCEPTUAL CONSTRAINTS
}

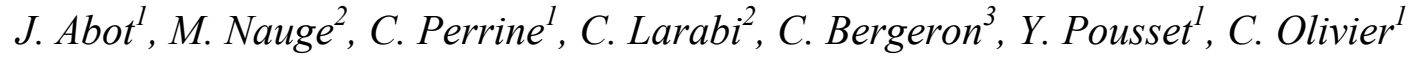 \\ XLIM-SIC CNRS Laboratory, SYSCOM ${ }^{1}$ and ICONES $^{2}$ teams, University of Poitiers, France \\ THALES Communications ${ }^{3}$, EDS/SPM, France
}

\begin{abstract}
This paper proposes a global approach of JPWL (ISO/IEC 15444-11) image transmission over a realistic wireless channel able to ensure the best Quality of Service (QoS). In order to exploit the channel diversity, we consider a ClosedLoop MIMO-OFDM scheme with different precoder designs. In particular, the high flexibility of QoS precoder allows taking into account the scalability of JPWL jointly with the instantaneous MIMO channel status. This increases the visual quality of received images. The monitoring of the quality is made by a reduced-reference metric (QIP) based on object's saliency and interest point, both linked to human perception. It is performed in association with a robust JPWL decoder to determine the optimal decoding configuration in terms of PSNR. The proposed scheme provides very good results and its performance is shown through a realistic wireless channel.
\end{abstract}

Index Terms-MIMO-OFDM, precoder design, interest points, reduced-reference metric, realistic wireless channel

\section{INTRODUCTION}

During the last decade, image transmissions over wireless channels became a very popular multimedia service in particular with the development of mobile devices (phone, tablets, etc). This implies the transmissions of images with continuously increasing quality, resolution and quantity like the High Definition (HD) technology. In addition, the wireless medium changes over time in an unpredictable way due to factors related to the mobility of both users and environments. The unstable nature and the limited bandwidth of wireless links are the key issues that must be taken into account to guarantee high quality multimedia services.

It is now proven that the usage of Multiple Input Multiple Output (MIMO) systems in a rich scattering environment significantly improves the reliability and/or the throughput of data transmission, in comparison to Single Input Single Output (SISO) systems [1]. So, in this paper, we focus on image transmission schemes based on Closed-Loop MIMO (CL-MIMO) systems. These latters exploit the Channel State Information (CSI) at the transmitter side to adjust the power reaching each antenna, known as UPA (Unequal Power Allocation), taking into account both the instantaneous channel status and the magnitude of the corresponding image codestream. We also use an OFDM (Orthogonal Frequency Division Multiplex) modulation, which is an efficient way to overcome frequency-selective fading due to multipath. The content to be transmitted is encoded using JPWL as specified in [2].

Different transmission schemes of the literature have shown the benefits of UPA strategies to transmit images. For instance, [3] proposes an UPA strategy based on a distortion model for JPEG image transmission over MIMO systems. Images are encoded into quality layers (a DC layer and 63 AC layer) grouped in bitstreams transmitted through a $4 \times 4$ MIMO system with a spatial multiplexing. Their model allows a significant gain in terms of PSNR but they consider error-free transmission of markers and headers, which is not the case with JPEG 2000 or JPWL transmission. In [4], the authors propose a CL-MIMO-OFDM scheme for transmission of JPEG 2000 images using the beamforming algorithm. Their approach consists in adding information about the SNR (Signal to Noise Ratio) of some subcarriers in a limited feedback to take into account clusters of unused subcarriers with conventional beamforming. However, the major part of transmission schemes from literature is not developed based on the content to be transmitted. These schemes try to adapt the content to ensure QoS. In addition, they do not use realistic channels to assess their performance but simplistic ones like Gaussian or Rayleigh statistic models. Finally, QoS is only considered like a perspective of transmission [SNR, capacity, Bit (BER) or Frame (FER) Error Rate] and does not reflect the subjective judgment of end-users. Indeed, as we shown in [5], the header protection predefined by JPWL standard can reduce cases of decoder crash but there are still cases where the decoder crash which is a problem for the QoS.

In this paper we propose a novel scheme for JPWL transmission over MIMO channel by using precoder solutions and by monitoring the layers' decoding. The first contribution of this work is to provide the power allocation, which minimizes the received image distortion regardless of the channel conditions. Indeed, we exploit the high flexibility in power allocation of QoS precoder [6] to finely adjust the power allocation by considering the magnitude of JPWL codestream and the channels status. The proposed QoS precoder solution is compared to the two other precoder designs: Maximum-SNR [7] and Water Filling [6] (WF). These latters allocate the transmitted power maximizing criteria like the SNR or the channel capacity. Those criteria, in addition to the channel conditions, influence the received JPWL quality over the precoder. The second contribution consists on using a realistic time varying MIMO channel to adapt the quality of the received image by the precoder solutions. To do this, we use a transmission channel based on a determinist model from a 3D ray tracer [8]. The topology of the used scene provides different transmission conditions alternating LOS (Line Of Sight) and NLOS (Non LOS) configuration. The third and last contribution of this paper lies on the use of human perception to ensure the best decoding. The Quality of Experience (QoE) of the end-user is estimated by a reduced-reference metric based on both visual saliency and interest points.

The remainder of this paper is organized as follows: In section 2, we describe the contributions of this paper through three major points: robust JPWL decoding, power allocation strategy and optimal decoding based on perception. Section 3 is devoted to the simulation of the complete chain and the discussion of the obtained results. Finally, this paper ends with some conclusions and future works.

\section{PROPOSED WORK}

\subsection{Global scheme}

We propose a global scheme taking into account a hierarchical content to be transmitted (Fig. 1). This combines the JPWL codec with precoder solutions. On the one hand, the JPWL generates $b$ codestreams sorted in a decreasing magnitude order. On the other hand, the precoder solutions decouple a MIMO channel into hierarchical, parallel and independent SISO sub-channels of different magnitudes and sorted in a decreasing SNR order. 


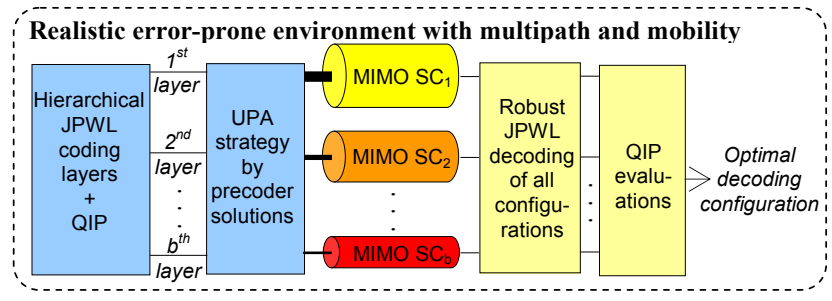

Figure 1: Proposed scheme based on content to be transmitted

Thus, the image transmission strategy adopted in this paper assigns each JPWL codestream to the corresponding SISO sub-channel providing a UPA strategy without any extra redundancy. The robustness of the scheme is ensured by the matching between the quality layers hierarchy and MIMO sub-channels hierarchy (MIMO SC in Fig. 1). The UPA strategy is applied to guarantee partial or total reception of the image depending on the channel status. During the JPWL coding process, a small amount of data ( 22 bytes), extracted from the original image and called reduced-reference is embedded in the codestream by the QIP metric described in the next sections. At the decoding side, this metric is jointly used with a robust JPWL decoder in order to provide the best decoding configuration to the user by exploiting the embedded information.

\subsection{UPA strategy}

The precoder solutions compute the coefficients according to both the channel status and the maximized criteria as mentioned previously. Thus, this strategy is adaptive to channel variations and the received image quality depends on the maximized criteria. The Max_SNR and WF optimize respectively the received SNR and the channel capacity. Hense, these precoder solutions do not really take into account the importance of the JPWL quality layer. However, the QoS precoder solution, which allows a high flexibility on the power allocation process, can be jointly adopted regarding the channel status and the magnitude of the JPWL quality layer transmitted over the $b$ SISO sub-channels. In this paper, we use an empirical approach to configure the QoS for the minimization of the received image distortion whatever the channel conditions.

\subsection{Robust JPWL decoder}

At the decoder side, the JPWL decoder should correct the received JPWL codestreams and rebuild the JPEG 2000 frames. So, in the implementation of this work, the JPWL decoder uses the EPBs (Error Protection Block) information to correct errors. But to prevent residual errors that could appear after RS (ReedSolomon) decoding, a robust implementation of JPWL is proposed, by saving on each step, the partial decoding of the image as shown on Fig. 2:

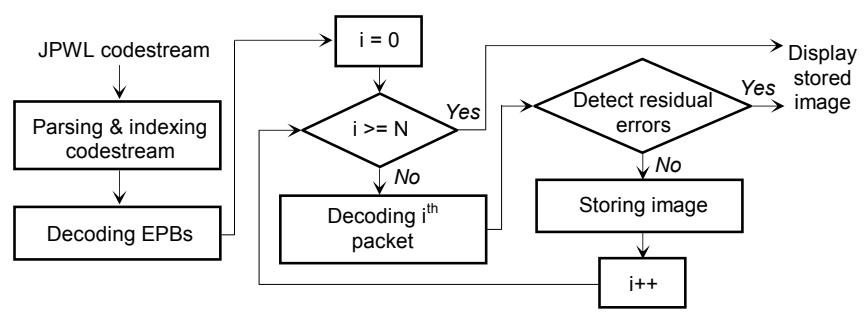

Figure 2: Implementation of the robust JPWL decoder

The procedure is described as follows:

- The JPWL codestream input is parsed and indexed to the $N^{\text {th }}$ packets JPEG 2000 codestream;

- This indexed codestream is transmitted to the JPWL decoder to use the redundancy data added in EPBs, and rebuilds the JPEG 2000 baseline codestream;
- Then for each JPEG 2000 packet, the packet is decoded:

- If the decoding succeeded, the partial decoding of the image is stored, and the next packet is processed;

- If the decoding process was unsuccessful, the global decoding process is aborted, and the partial decoding is provided as output.

\subsection{Decoding strategy with QIP}

We used a quality metric able to capture the perceived quality of an image. However, in the transmission context, the original image is not available at the receiver. Hence, pixel-wise comparisons as applied by full-reference metrics (like PSNR or SSIM) are not possible and no-reference metrics assume that the noise is known and easily measurable. So, the best way, in our case, is the use of a reduced-reference metric. This strategy allows to exploit a small part of the reference for quality measurement purposes.

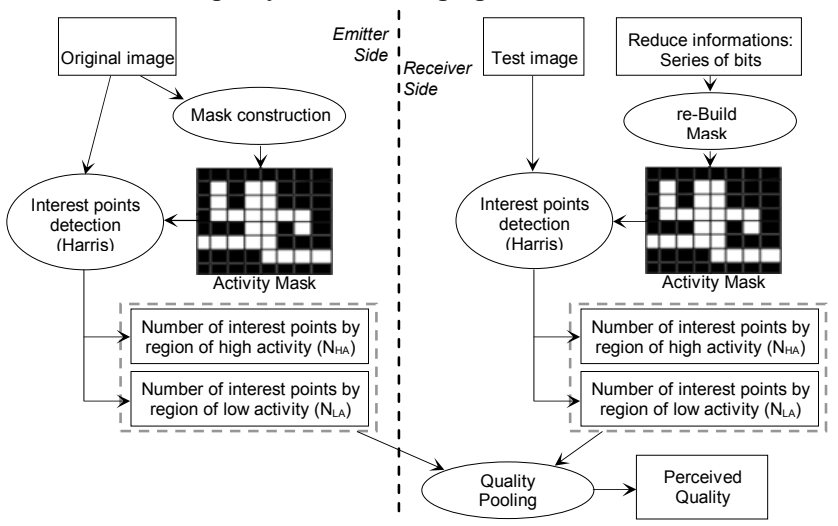

Figure 3: Flowchart of the QIP reduced-reference metric

QIP [9] belongs to the last category. It is based on the ability of the interest points to predict a variation in the image, depending on the object's saliency. As described in Fig. 3, the metric decomposes the original image into $12 \times 12$ equal partitions on which Harris interest points detector is applied. Each partition is classified in terms of saliency (HA: High Activity or LA: Low Activity) and its number of interest points is added to the final number ( $N_{H A}$ or $N_{L A}$ ). So the reduced-reference to be added to the transmitted image is composed of 2 integers $\left(N_{H A}\right.$ and $\left.N_{L A}\right)$ and 18 bytes representing the activity mask $(12 \times 12$ bits). The same procedure is reproduced at the receiver by exploiting the received activity mask. The perceived quality is computed by comparing the received and computed numbers of interest points. QIP is used in the proposed work as a layer selector able to detect a reduction of the perceived quality while decoding an additional layer. In our strategy, we decode all the $b$ possible configurations with JPWL robust decoder. Each configuration is evaluated by QIP which assigns a score between 0 (very bad quality) and 1 (excellent quality). Finally, we retain the configuration having the best QIP score that corresponds to the best Quality of Experience (QoE).

\section{SIMULATION RESULTS}

This section is dedicated to the simulation of the whole proposed scheme by testing each part of it and giving enough evidence about the interest of the proposed work. We consider the transmission of JPWL codestream over a $3 \times 3$ MIMO system that leads to consider 3 different SISO sub-channels. Thus, the strategy adapts the transmission of 1 to 3 JPWL quality layers. In reception, the 22 bytes of the QIP reduced-reference is considered error free.

\subsection{JPWL configuration}

We consider equivalent source rates on each virtual sub-channel. The source rates constraint correspond to the diagram given below: 


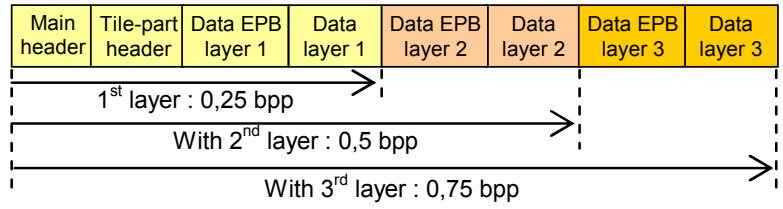

Figure 4: Layers and EPB data repartitions with JPWL coding bitrates

The coding bitrates (Fig. 4) offer a good compromise between acceptable basic quality and enough quality gain between the different layers. To limit transmission errors, we use the following tools of JPWL:

- SOP (Start Of Packet) and EPH (End of Packet Header) resynchronization markers;

- Main header protection and tile-part header protection predefined by the JPWL standard;

- $\mathrm{RS}(38,32)$ codes on data layer (Fig. 4).

Thanks to UPA, we can reduce the redundancy of ECC (Error Correcting Codes). Accordingly, we use ECC with high coding rate in an EEP (Equal Error Protection) strategy to overcome residual errors. For simulations, we consider two configurations. The first one does not use $\mathrm{RS}(38,32)$ codes to strengthen the impact of QIP metric on the decoding process. The second one considers an EEP strategy that takes into account $\operatorname{RS}(38,32)$ codes. This configuration is used to compare the overall performance of the UPA strategies proposed by the different precoder solutions.

We use the image "caps" for simulations of transmission. The table below summarizes the scores in terms of PSNR (in $\mathrm{dB}$ ) and QIP corresponding to the encoding configurations:

Table 1: Characteristics of image "Caps" used for simulation

\begin{tabular}{c|cc|cc|cc}
\hline & \multicolumn{2}{|c|}{$1^{\text {st }}$ layer } & \multicolumn{2}{c|}{ With 2 $^{\text {nd }}$} & layer & \multicolumn{2}{c}{ With $3^{\text {rd }}$ layer } \\
& PSNR (dB) & QIP & PSNR (dB) & QIP & PSNR (dB) & QIP \\
\hline no ECC & 31,18 & 0,905 & 34,85 & 0,976 & 37,14 & 0,975 \\
EEP & 30,33 & 0,857 & 33,86 & 0,956 & 36,08 & 0,993 \\
\hline
\end{tabular}

\subsection{Realistic error-prone environment}

We use a realistic transmission channel for simulations (Fig. 5):

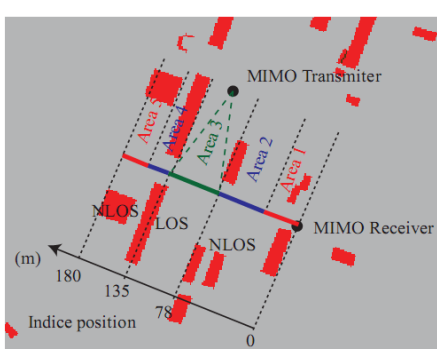

(a)

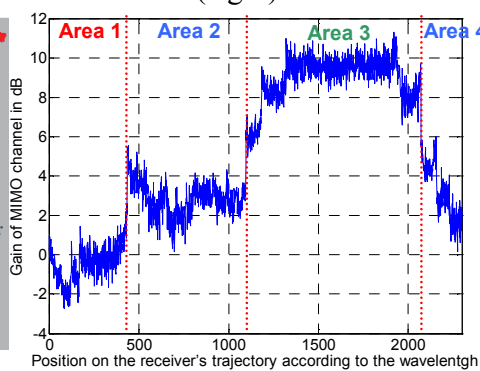

(b)
Figure 5: (a) Topology of the transmission scene and (b) gain evolution of the MIMO channel

The Impulse Responses (IRs) are provided by a channel simulator which is based on a 3D ray tracer [8]. The used scene is a sub-urban environment presented in Fig. 5-(a) where the buildings are in red. The MIMO transmitter is fixed and the MIMO receiver moves throughout a distance of $180 \mathrm{~m}$ at a given speed, while the transmission can be bad (NLOS in area 1), average (NLOS in areas 2 and 4 ) or good (LOS in area 3 ). The gain evolution of the MIMO channel is presented in the Fig. 5-(b).

\subsection{QoS precoder configuration}

During the precoding step, the precoder applies a Singular Value Decomposition (SVD) on the channel matrix that leads to consider a virtual channel matrix $H_{v}$ described as:

$$
H_{v}=\operatorname{diag}\left(\sigma_{1}, \sigma_{2}, \ldots, \sigma_{b}\right)
$$

The Eigen-value $\sigma_{i}^{2}$ represents the SNR value of the SISO sub-channel $i$. After the precoding step, the SNR of this latter becomes equal to $\sigma_{i}^{2} f_{i}^{2}$ instead of $\sigma_{i}^{2}$ where $f_{i}$ are the precoder coefficients computed according to both channel status and the maximized criteria described previously. In our scheme, we consider an average using based on the content to be transmitted for the QoS precoder on each areas of the realistic channel. Thus we solve directly $f_{i}^{2}$ as given in Table 2:

\section{Table 2: Three considered configurations for the QoS precoder}

\begin{tabular}{cccc}
\hline Channel conditions & Bad & Average & Good \\
Transmission area & Area 1 & Areas 2 \& 4 & Area 3 \\
$\boldsymbol{f}_{\boldsymbol{i}}{ }^{2}$ values & {$\left[\begin{array}{lll}1 & 0 & 0\end{array}\right]$} & {$\left[\begin{array}{lll}0,4 & 0,6 & 0\end{array}\right]$} & {$\left[\begin{array}{lll}0,05 & 0,15 & 0,8\end{array}\right]$} \\
\hline
\end{tabular}

This setting was defined to ensure the best visual quality. This system guarantees the correct transmission of the $1^{\text {st }}$ quality layer in bad conditions (area 1). It adds the $2^{\text {nd }}$ quality layer when conditions become medium (areas 2 and 4). Finally, it transmits all the layers in good conditions (area 3).

\subsection{Transmission chain}

The transmission chain includes specific parameters of 802.11n standard [10]. We do not use ECC provided by this standard, but we use the ECC provided by JPWL. We use 4-QAM (Quadrature Amplitude Modulation), various precoder design solutions and a Maximum Likelihood (ML) decoder. The mobility in the scene is set at a speed of $5 \mathrm{~m} / \mathrm{s}$. The CSI is known at both the transmitter and receiver sides. We perform new channel estimation every 300 OFDM symbols, which may overcome the Doppler effect. The system achieves an overall transmission rate of $24 \mathrm{Mbits} / \mathrm{s}$.

\subsection{Benefits of using QIP metric}

We illustrate the QIP efficiency with EPA (Equal Power Allocation) configuration (Fig. 6). The QIP effect on the decoding process is highlighted by not using ECC.

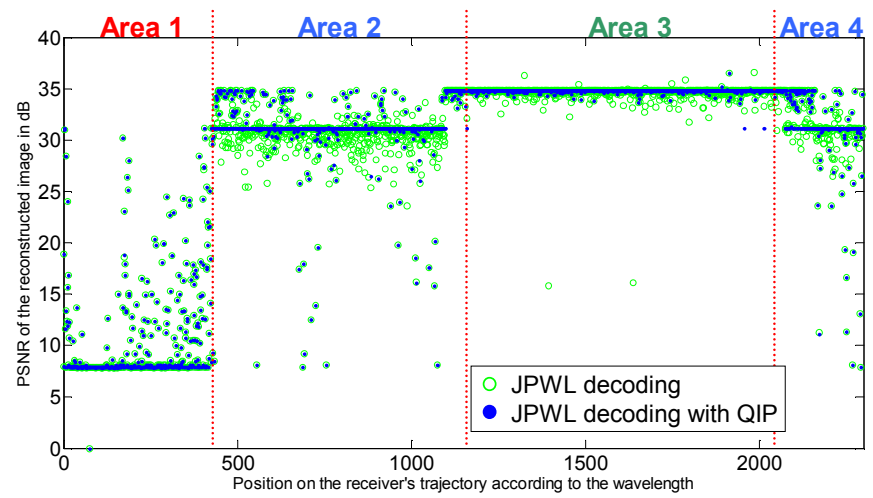

Figure 6: Variation of the PSNR function of the decoding mode "caps"; EPA configuration

Fig. 6 illustrates the effect of the QIP metric on the decoding process. Each point represents the PSNR of a decoded image along the trajectory. We compare two decoding modes: the conventional decoding proposed by JPWL and the JPWL decoding associated with QIP. We can notice that the conventional decoding method is suboptimal because the decoder can decode quality layers responsible for errors and thus decreases the overall quality as shown in Fig. 7.

QIP is able to detect a variation of the number of interest points, which indicates an image distortion due to transmission errors. Thus, this metric is able to provide, in a realistic way, the decoding configuration providing the minimum visual errors. We summarize the QIP performance by statistic results in Table 3. 


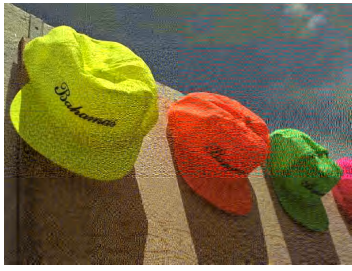

(a) 2 quality layers with JPWL

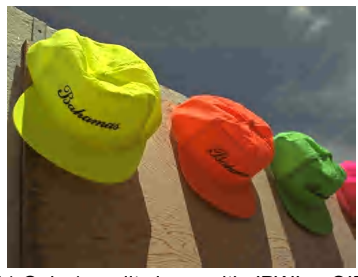

(b) Only 1 quality layer with JPWL + QIP

(b) Only 1 quality layer with
t obtained by using QIP;

$R=23,62 d B-Q I P=0,135$; (b) $P S N R=31,18 d B-Q I P=0,905$
Table 3: Statistics on performance of OIP metric

\begin{tabular}{c|cc|cc|cc}
\hline $\begin{array}{c}\text { JPWL } \\
\text { decoding } \\
\text { strategy }\end{array}$ & \multicolumn{2}{|c|}{ Bad conditions } & \multicolumn{2}{c|}{ Average conditions } & \multicolumn{2}{c}{ Good conditions } \\
\hline $\begin{array}{c}\text { PSNR } \\
\text { maximization }\end{array}$ & $98,39 \%$ & $99,77 \%$ & $52,37 \%$ & $89,28 \%$ & $64,82 \%$ & $98,57 \%$ \\
$\begin{array}{c}\text { Best decoding } \\
\text { strategy }\end{array}$ & $0,23 \%$ & $1,61 \%$ & $10,72 \%$ & $47,63 \%$ & $1,43 \%$ & $35,18 \%$ \\
$\begin{array}{c}\text { Average } \\
\text { PSNR }\end{array}$ & $10,82 \mathrm{~dB}$ & $10,82 \mathrm{~dB}$ & $30,64 \mathrm{~dB}$ & $31,09 \mathrm{~dB}$ & $34,66 \mathrm{~dB}$ & $34,82 \mathrm{~dB}$ \\
\hline
\end{tabular}

This table shows the contribution of QIP into the decoding process. For instance, for average conditions of transmission, QIP provides the best score in terms of PSNR in $89,28 \%$ of cases and improves results in $47,63 \%$ of cases. The lack of decoding strategy leads obviously to decode more layers despite the bad visual quality. Optimal configurations are achieved by using a perceptually-inspired metric like QIP.

\subsection{Performance of precoder solutions}

In this section, we compare the performance of the different precoding solutions by using an EEP strategy (Tab. 1). To make the curves more readable, we averaged them with a sliding window containing 20 values (Fig. 8):

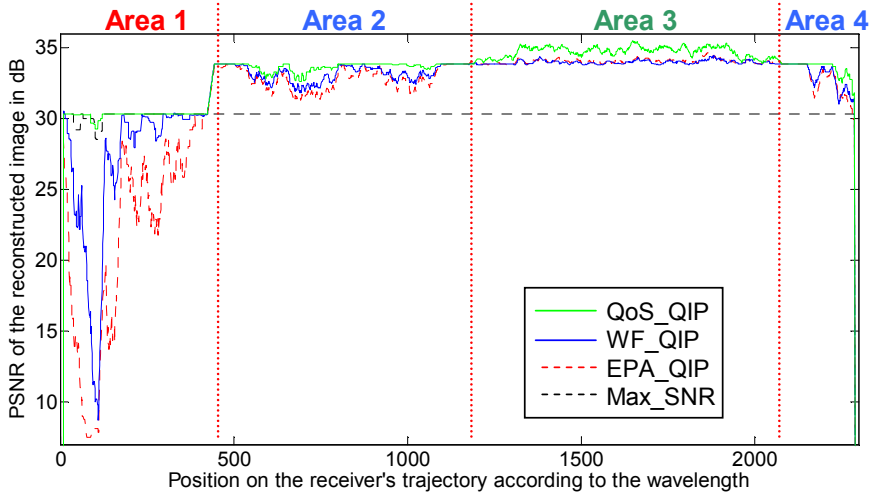

Figure 8: PSNR variation according to the precoding solutions

On this curve, we show the PSNR variation of the reconstructed images (y-axis) versus position on the receiver's trajectory (x-axis) for the different precoding solutions. For bad conditions (area 1), it is not possible to transmit 2 quality layers without transmission errors. Thus, the most effective strategy is to put the full power on the $1^{\text {st }}$ sub-channel (QoS precoder and Max_SNR) to guaranty the $1^{\text {st }}$ quality layer. So, it is not possible to obtain better results on this area. WF maximizes the channel capacity. Hence, it allocates power on the $2^{\text {nd }}$ sub-channel, which reduces the performance in terms of visual quality. With average conditions (areas 2 and 4), WF allocates power on all subchannels while QoS allocates power to ensure only 2 quality layers without error. This can be explained by the fact that the $3^{\text {rd }}$ quality layer is never de- codable without error. Max_SNR transmits only the $1^{\text {st }}$ layer what explains it does not exceed the PSNR related to this one. Finally when there are good conditions (area 3), the WF behavior tends to an EPA strategy, which does not ensure the $3^{\text {rd }}$ layer error-free transmission. The QoS precoder takes advantage of high SNR values on $1^{\text {st }}$ and $2^{\text {nd }}$ sub-channels to provide the maximum power on the $3^{\text {rd }}$ sub-channel. That drastically reduces the BER on the $3^{\text {rd }}$ quality layer what explains the better behavior of this precoding solution. Thus, we can see that taking into account the content improves the overall quality of received images whatever the channel conditions.

\section{CONCLUSION \& FUTURE WORKS}

In this paper, we proposed a transmission scheme taking into account the overall content to ensure QoS. To do this, we take advantage of the channel transmission by exploiting the spatial diversity offered by the MIMO channel and its decomposition into virtual SISO sub-channels. These latter are suitable for an image coded into a hierarchical quality layer by a JPWL encoder. We also proposed a decoding strategy taking into account a reduced-reference metric named QIP, based on human perception, and a robust JPWL decoder. This decoding strategy can solve cases where an additional quality layer can lead to distortions due to residual errors. This ensures the best decoding configuration. We showed that taking into account the content allows a significant gain in quality and robustness.

As future works, we consider the optimization of the QoS precoder settings by an adaptive algorithm providing the best QoE. Accordingly we would take into account the instantaneous channel status rather than an average behavior. Thus the results could be improved. We can also consider the adequacy of this strategy with conventional UEP (Unequal Error Protection) strategy that would lead to even greater robustness. An extension of QIP by using a hierarchical saliency map can be envisaged in addition to extensive psychovisual experiments for QoE validation.

\section{ACKNOWLEDGMENT}

This work has received support from the French National Research Agency through the CAIMAN project, ANR-08-TO-002.

\section{REFERENCES}

[1] I.Telatar, "Capacity of multi-antennas Gaussian channels", Eur, Trans. Telecomm. ETT, vol.10, no.6, pp. 585-595, Nov. 1999.

[2] ISO/IEC 15444-11:2007, "JPEG2000 image coding system - part 11: Wireless JPEG2000", May 2007.

[3] M.F. Sabir, A.C. Bovik, R.W. Heath, Jr., 'Unequal Power Allocation for JPEG Transmission Over MIMO Systems", IEEE Transactions on image processing, vol. 19, No. 2, Feb 2010

[4] U. Sethakaset, S. Sumei, "Robust JPEG2000 Image Transmission over ClosedLoop MIMO-OFDM with Limited Feedback", IEEE PIMRC 2008, Cannes, France.

[5] D. Nicholson, C. Lamy-Bergot, X. Naturel, and C. Poulliat, "JPEG2000 backward compatible error protection with Reed Solomon codes", IEEE Trans. On Consumer Electronics, vol. 49, no. 4, pp. 855-860, Nov. 2003.

[6] H. Sampath, P. Stoica, A. Paulraj, "Generalized Linear Precoder and Decoder Design for MIMO Channels Using the Weighted MMSE Criterion", IEEE Trans. Commun., Vol. 49, No. 12, pp. 2198-2206, Dec. 2001.

[7] P. Stoica, G. Ganesan, "Maximum-SNR spatial-temporal formatting designs for MIMO channels", IEEE Trans. Signal Processing, vol. 50, No. 12, pp. 3036-3042, Dec. 2002.

[8] Y. Chartois, Y. Pousset, R. Vauzelle, "A SISO and MIMO radio channel characterization with a 3D ray tracing propagation model in urban environment', European Conference on Propagation and Systems, March 2005.

[9] M. Nauge, M.-C. Larabi and C. Fernandez, "A reduced-reference metric based on the interest points in color images”, PCS'2010, pp. 610-613, Nagoya, Japan.

[10] IEEE Standard for Information Technology-Part 11: Wireless LAN Medium Access Control (MAC) and Physical Layer (PHY) specifications Amendment: Enhancements for Higher Throughput (802.11n), 2009. 